\title{
Algebraic Structures Based on a Classifying Space of a Compact Lie Group
}

\author{
Dae-Woong Lee \\ Department of Mathematics and Institute of Pure and Applied Mathematics, Chonbuk National University, 567 Baekje-daero, \\ Deokjin-gu, Jeonju-si, Jeollabuk-do 561-756, Republic of Korea \\ Correspondence should be addressed to Dae-Woong Lee; dwlee@jbnu.ac.kr
}

Received 5 July 2013; Revised 10 September 2013; Accepted 20 September 2013

Academic Editor: Teoman Özer

Copyright (C) 2013 Dae-Woong Lee. This is an open access article distributed under the Creative Commons Attribution License, which permits unrestricted use, distribution, and reproduction in any medium, provided the original work is properly cited.

We analyze the algebraic structures based on a classifying space of a compact Lie group. We construct the connected graded free Lie algebra structure by considering the rationally nontrivial indecomposable and decomposable generators of homotopy groups and the cohomology cup products, and we show that the homomorphic image of homology generators can be expressed in terms of the Lie brackets in rational homology. By using the Milnor-Moore theorem, we also investigate the concrete primitive elements in the Pontrjagin algebra.

\section{Introduction}

A Lie group is a differentiable manifold $M$ with a group structure in which the multiplication $M \times M \rightarrow M$ and the inversive map $M \rightarrow M\left(m \mapsto m^{-1}\right)$ are differentiable. Therefore, it can be studied using differential calculus in contrast with the case of more general topological groups as a special case of $\mathrm{H}$-spaces. It is well known that the only spheres that are connected $\mathrm{H}$-spaces are $S^{1}, S^{3}$, and $S^{7}$. We note that the first two spheres are Lie groups while the last one is just an $\mathrm{H}$-space which is not an $A_{3}$-space but just an $A_{2}$-space in the sense of Stasheff [1]. Lie groups play an enormous role in algebraic topology as well as modern differential geometry on several different levels. The presence of continuous symmetries expressed via a Lie group action on a manifold places strong constraints on its geometry and facilitates analysis on the manifold. Moreover, linear actions of Lie groups are especially important and are studied in representation theory.

As usual we let $\Sigma$ and $\Omega$ be the suspension and loop functors in the (pointed) homotopy category, respectively. It is well known that the functors $\Sigma$ and $\Omega$ are examples of adjoint functors. Moreover, co- $\mathrm{H}$-spaces and $\mathrm{H}$-spaces are important objects of research in homotopy theory and they are the dual notions in the sense of Eckmann and Hilton. We refer to Arkowitz's paper [2] and Scheerer's article [3] for a survey of the vast literature about co-H-spaces, $\mathrm{H}$-spaces, and related topics.

Let $\operatorname{SNT}(X)$ denote the set of all homotopy types $[Y]$ such that $X$ and $Y$ have the same $n$-type for each nonnegative integer $n$ (see [4-6]). McGibbon and Møller [7] showed that if $G$ is a connected compact Lie group, then its classifying space usually has an uncountable $S N T(B G)$ except for several cases and gave an excellent set of examples. Furthermore, in [8] the classical projective $n$-spaces (real, complex, and quaternionic) were studied in terms of their self-maps from a homotopy point of view. Recently, some common fixed point results for single as well as set valued mappings involving certain rational expressions in complete partial metric spaces were obtained in [9]. Moreover, some fixed point and common fixed point theorems on ordered cone $b$-metric spaces were also established in [10].

In this paper all spaces are based and have the based homotopy type of based, connected CW-complexes. All maps and homotopies preserve the base point. Unless otherwise stated, we do not distinguish notationally between a map and its homotopy class.

The main purpose of this paper is to investigate the algebraic explanation based on a classifying space of the compact Lie group $U(1)$. After constructing self-maps using the suspension structure, we define a useful commutator of selfmaps on the suspension of a classifying space of the compact 
Lie group. We construct the connected graded free Lie algebra structure by considering the rationally nontrivial indecomposable and decomposable generators of homotopy groups and the cohomology cup products. We show that the homomorphic image of homology generators can be expressed in terms of the Lie brackets in rational homology. By using the Milnor-Moore theorem, we also investigate the concrete primitive elements as the images of the Hurewicz homomorphisms in the Pontrjagin algebra.

\section{Preliminaries}

Let $\mathbb{Z}, \mathbb{Q}, \mathbb{R}$, and $\mathbb{C}$ denote the ring of integers, the fields of rational, real, and complex numbers, respectively. The unitary group $U(n)$ is the group of $n \times n$ unitary matrices with the group operation that of matrix multiplication as a subgroup of the general linear group $\mathrm{GL}(n, \mathbb{C})$. The unitary group $U(n)$ is a real Lie group of dimension $n^{2}$. The Lie algebra of $U(n)$ consists of $n \times n$ skew-Hermitian matrices with the Lie bracket given by the commutator. The first unitary group $U(1)$ is canonically the circle group consisting of all complex numbers with absolute value 1 under multiplication; that is, the set of all $1 \times 1$ unitary matrices. The first unitary group shows up in a variety of forms in mathematics. Some of the more common forms are as follows:

$$
U(1) \cong T \cong \mathbb{R} / \mathbb{Z} \cong \mathrm{SO}(2)
$$

where $T$ is a one torus and $\mathrm{SO}(2)$ is the orthogonal $2 \times 2$ matrices with determinant 1 . The latter is said to be the special orthogonal group.

A classifying space $B G$ of a topological group $G$ is the quotient of a weakly contractible space $E G$ by a free action of $G$. It has the property that any principal $G$-bundle over a paracompact manifold is isomorphic to a pullback of the principal bundle $E G \rightarrow B G$. We note that the classifying space functor $B$ is essentially inverse to the loop space functor $\Omega$ in algebraic topology.

If $X$ is a co-H-group with comultiplication

$$
\varphi: X \longrightarrow X \vee X
$$

and homotopy inverse, then for every pointed space $Y$ the set $[X, Y]$ of homotopy classes from $X$ to $Y$ can be given the structure of a group under the addition as follows: for $a, b \in[X, Y]$, the binary operation, denoted " $a+b$ ", is defined by the homotopy class of the composition of maps

$$
X \stackrel{\varphi}{\rightarrow} X \vee X \stackrel{a \vee b}{\longrightarrow} Y \vee Y \stackrel{\nabla}{\rightarrow} Y,
$$

where $\nabla$ is a folding map.

The Eckmann-Hilton dual of a co-H-group is an $\mathrm{H}$-group (see $[11,12])$. As an adjointness, if $X$ is any pointed space and $(Y, m)$ is an $H$-group, then the set $[X, Y]$ becomes a group if we define the product " $c \cdot d$ " to be the homotopy class of the composition of maps

$$
X \stackrel{\Delta}{\rightarrow} X \times X \stackrel{c \times d}{\longrightarrow} Y \times Y \stackrel{m}{\rightarrow} Y,
$$

where $\Delta: X \rightarrow X \times X$ is the diagonal map, $c, d \in[X, Y]$, and $m: X \times X \rightarrow X$ is a multiplication.

The principle examples of a co-H-group and an $\mathrm{H}$-group are the suspension $\Sigma X$ and the loop space $\Omega X$ of a space $X$, respectively.

We note that $B U(1)$ has a $\mathrm{CW}$-decomposition as follows:

$$
B U(1)=S^{2} \cup_{\gamma_{1}} e^{4} \cup_{\gamma_{2}} e^{6} \cup_{\gamma_{3}} \cdots \cup_{\gamma_{n-1}} e^{2 n} \cup_{\gamma_{n}} e^{2(n+1)} \cup_{\gamma_{n+1}} \cdots,
$$

where $\gamma_{n}$ is an attaching map for $n=1,2,3, \ldots$.

From now on, we denote $B U(1)_{k}$ by the $k$-skeleton of a $\mathrm{CW}$-complex $B U(1)$. We define maps $f_{n}: B U(1) \rightarrow \Omega \Sigma B U(1)$ for $n=1,2,3, \ldots$ as follows.

Definition 1. The cofibration sequence

$$
B U(1)_{2 n-1} \stackrel{i_{n}}{\longleftrightarrow} B U(1) \stackrel{p_{n}}{\longrightarrow} \frac{B U(1)}{B U(1)_{2 n-1}}
$$

induces an exact sequence of groups

$$
\begin{aligned}
{\left[\frac{B U(1)}{B U(1)_{2 n-1}}, \Omega \Sigma B U(1)\right] } & \stackrel{p_{n}^{\sharp}}{\longrightarrow}[B U(1), \Omega \Sigma B U(1)] \\
& \stackrel{i_{n}^{\sharp}}{\longrightarrow}\left[B U(1)_{2 n-1}, \Omega \Sigma B U(1)\right],
\end{aligned}
$$

for each $n=1,2,3, \ldots$. We now take an essential map

$$
f_{n} \in i_{n}^{\#^{-1}}(*)=\operatorname{ker}\left(i_{n}^{\sharp}\right) \subset[B U(1), \Omega \Sigma B U(1)],
$$

for each $n=1,2,3, \ldots$. Similarly, by using the above exact sequences, we can choose an essential map

$$
\tilde{f}_{n}: \frac{B U(1)}{B U(1)_{2 n-1}} \longrightarrow \Omega \Sigma B U(1),
$$

such that $p_{n}^{\sharp}\left(\tilde{f}_{n}\right)=f_{n}$ for $n=1,2,3, \ldots$.

In the above definition, we note that $B U(1)_{1}=*$, and thus $p_{1}^{\sharp}$ is an isomorphism. We also note that

$$
\frac{B U(1)}{B U(1)_{2 n-1}}=S^{2 n} \cup \text { higher cells. }
$$

We now define the following.

Definition 2. We define a rationally nontrivial homotopy element

$$
\xi_{n}: S^{2 n} \longrightarrow \Omega \Sigma B U(1)
$$

of the homotopy groups modulo torsions $\pi_{2 n}(\Omega \Sigma B U(1)) /$ torsion by $\xi_{n}=\left.\widetilde{f}_{n}\right|_{S^{2 n}}$ for each $n=1,2,3, \ldots$

Now we take the self-map

$$
F_{n}: \Sigma B U(1) \longrightarrow \Sigma B U(1)
$$

by the adjointness of

$$
f_{n}: B U(1) \longrightarrow \Omega \Sigma B U(1)
$$

for each $n=1,2,3, \ldots$. 
We recall that

$$
\widetilde{H}_{*}(B U(1) ; \mathbb{Q}) \cong \mathbb{Q}\left\{u_{1}, u_{2}, \ldots, u_{n}, \ldots\right\}
$$

as a graded $\mathbb{Q}$-module, where $u_{n}$ is the standard generator of $H_{2 n}(B U(1) ; \mathbb{Q})$ for each $n=1,2,3, \ldots$. The salient BottSamelson theorem [13] says that the Pontrjagin algebra $H_{*}(\Omega \Sigma B U(1) ; \mathbb{Q})$ is isomorphic to the tensor algebra $T H_{*}(B U(1) ; \mathbb{Q})$. In other words, the rational homology of $\Omega \Sigma K$ is the tensor algebra $T\left\{u_{1}, u_{2}, \ldots, u_{n}, \ldots\right\}$ generated by $\left\{u_{1}, u_{2}, \ldots, u_{n}, \ldots\right\}$, where $u_{n}$ is a rational homology generator with diagonal

$$
\Delta\left(u_{k}\right)=\sum_{i+j=k} u_{i} \otimes u_{j}
$$

and $u_{n}=E_{*}\left(u_{n}\right)$. Here, $E$ is the canonical inclusion map $E: B U(1) \rightarrow \Omega \Sigma B U(1)$ defined by $E(s)(t)=\langle s, t\rangle \epsilon$ $\Sigma B U(1)$, and $u_{0}$ means 1 . From the Serre spectral sequence of a fibration $\Omega B U(1) \rightarrow P B U(1) \rightarrow B U(1)$, we have an algebra isomorphism

$$
H^{*}(B U(1) ; \mathbb{Q}) \cong \mathbb{Q}[\alpha] .
$$

Here $\mathbb{Q}[\alpha]$ is the polynomial algebra over $\mathbb{Q}$ generated by $\alpha$ of degree 2; that is, $\alpha$ is a generator of $H^{2}(B U(1) ; \mathbb{Q})$ with the Kronecker index $\left\langle\alpha^{i}, u_{j}\right\rangle=\delta_{i j}$.

We now consider a wedge of spheres $X=S^{n_{1}} \vee S^{n_{2}} \vee \cdots \vee S^{n_{k}}$ for $2 \leq n_{1} \leq n_{2} \leq \cdots \leq n_{k}$. Let $r_{t}: S^{n_{t}} \rightarrow X$ be the $t$ th inclusion for $t=1,2, \ldots, k$. We then inductively define and order basic (Whitehead) products as follows. Basic products of weight 1 are (in order) $r_{1}, r_{2}, \ldots, r_{k}$. Assume basic products of weight $<n$ have been defined and ordered so that if $r<s<n$, any basic product of weight $r$ is less than all basic products of weight $s$. Then a basic product of weight $n$ is a Whitehead product $[a, b]$, where $a$ is a basic product of weight $m$ and $b$ is a basic product of weight $l, m+l=n, a<b$. Furthermore, if $b$ is a Whitehead product $[c, d]$ of basic products $c$ and $d$, then we require that $c \leq a$. The basic products of weight $n$ are ordered arbitrarily among themselves and are greater than any basic product of weight $<n$. Note that to a basic product of weight $n$ we can associate a string of distinct symbols $r_{v_{1}}, \ldots, r_{v_{s}}$, for $1 \leq v_{i} \leq k$, which are the elements which appear in the basic products. Suppose in the basic product $w_{s}, r_{p}$ occurs $l_{p}$ times, $l_{p} \geq 1$. Then the height of the basic product is $\sum l_{p}\left(r_{p}-1\right)+1$ and the length is $\sum l_{p}-1$. Clearly if $w_{s}$ has height $h_{s}$, then $w_{s} \in \pi_{h_{s}}(X)$. [14].

We end this section with the following Hilton's formula

Theorem 3. Let the ordered basic products of $X=S^{n_{1}} \vee S^{n_{2}} \vee$ $\cdots \vee S^{n_{k}}$ be $w_{1}, w_{2}, \ldots, w_{s}, \ldots$ with the height of $w_{s}=h_{s}$. Then for every $m$,

$$
\pi_{m}(X) \approx \bigoplus_{s=1}^{\infty} \pi_{m}\left(S^{h_{s}}\right) .
$$

The isomorphism $\theta: \bigoplus_{s=1}^{\infty} \pi_{m}\left(S^{h_{s}}\right) \rightarrow \pi_{m}(X)$ is defined by

$$
\theta \mid \pi_{m}\left(S^{h_{s}}\right)=w_{s *}: \pi_{m}\left(S^{h_{s}}\right) \longrightarrow \pi_{m}(X) .
$$

We note that the direct sum is finite for each $m$ since $h_{s} \rightarrow$ $\infty$.

\section{Commutators and Lie Algebra Structures}

By using the addition of a co-H-group in Section 2, we define the following.

Definition 4. We define a commutator

$$
C\left(F_{i_{1}}, F_{i_{2}}\right): \Sigma B U(1) \longrightarrow \Sigma B U(1)
$$

of $F_{i_{1}}$ and $F_{i_{2}}$ in $[\Sigma B U(1), \Sigma B U(1)]$ by

$$
C\left(F_{i_{1}}, F_{i_{2}}\right)=F_{i_{1}}+F_{i_{2}}-F_{i_{1}}-F_{i_{2}},
$$

where the operations are the suspension additions on $\Sigma B U(1)$, and

$$
-F: \Sigma B U(1) \longrightarrow \Sigma B U(1)
$$

is the suspension inverse defined by

$$
-F(\langle s, t\rangle)=F(\langle s, 1-t\rangle)
$$

for $s \in B U(1)$ and $t \in I$.

Let

$$
l: \Omega \Sigma B U(1) \longrightarrow \Omega \Sigma B U(1)
$$

be the map of loop inverse given by $l(\omega)=\omega^{-1}$, where $\omega^{-1}(t)=$ $\omega(1-t), t \in I$.

Similarly, we define the following.

Definition 5. The map $C\left(f_{i_{1}}, f_{i_{2}}\right): B U(1) \rightarrow \Omega \Sigma B U(1)$ is a commutator of $f_{i_{1}}$ and $f_{i_{2}}$ in $[B U(1), \Omega \Sigma B U(1)]$ defined by

$$
C\left(f_{i_{1}}, f_{i_{2}}\right)(s)=f_{i_{1}}(s) \cdot f_{i_{2}}(s) \cdot l\left(f_{i_{1}}(s)\right) \cdot l\left(f_{i_{2}}(s)\right),
$$

where $s \in B U(1)$, and the multiplication is the loop multiplication.

Remark 6. Let $c\left(f_{i_{1}}, f_{i_{2}}\right): B U(1) \times B U(1) \rightarrow \Omega \Sigma B U(1)$ be a map given by

$$
c\left(f_{i_{1}}, f_{i_{2}}\right)(s, t)=f_{i_{1}}(s) \cdot f_{i_{2}}(t) \cdot l\left(f_{i_{1}}(s)\right) \cdot l\left(f_{i_{2}}(t)\right) .
$$

Then we get

$$
C\left(f_{i_{1}}, f_{i_{2}}\right)=c\left(f_{i_{1}}, f_{i_{2}}\right) \circ \Delta,
$$

where $\Delta: B U(1) \rightarrow B U(1) \times B U(1)$ is the diagonal map.

Note that the weak category of $\Omega \Sigma B U(1)$ is not finite because there are infinitely many nonzero cohomology cup products in it, and thus it has the infinite LusternikSchnirelmann category [12, Chapter X]. Moreover, Arkowitz and Curjel [15, Theorem 5] showed that the $n$-fold commutator is of finite order if and only if all $n$-fold cup products of any positive dimensional rational cohomology classes of a space vanish (see also [16]). Therefore, we can consider the iterated commutators which are nontrivial in $\Omega \Sigma B U(1)$. 
We recall that the Samelson product gives $\pi_{*}(\Omega X), * \geq$ 1 , the structure of graded Lie algebra (see $[17,18]$ ); that is, if $x \in \pi_{p}(\Omega X), y \in \pi_{q}(\Omega X)$ and $z \in \pi_{r}(\Omega X)$, then

$$
\begin{gathered}
\langle x, y\rangle=(-1)^{p(q-1)}\langle y, x\rangle, \\
(-1)^{p r}\langle x,\langle y, z\rangle\rangle+(-1)^{p q}\langle y,\langle z, x\rangle\rangle \\
+(-1)^{q r}\langle z,\langle x, y\rangle\rangle=0 .
\end{gathered}
$$

Let $k_{1}: X \rightarrow X \times X$ and $k_{2}: X \rightarrow X \times X$ be the first and second inclusions between based spaces, respectively; that is, $k_{1}(x)=\left(x, x_{0}\right)$ and $k_{2}(x)=\left(x_{0}, x\right)$, where $x_{0}$ is the base point of $X$. Recall that an element $z \in H_{*}(X)$ is said to be primitive if and only if $\Delta_{*}(z)=k_{1 *}(z)+k_{2 *}(z)=z \otimes 1+1 \otimes z$ in homology, where $\Delta: X \rightarrow X \times X$ is the diagonal map.

Let $h: \pi_{*}(\Omega X) \rightarrow H_{*}(\Omega X ; \mathbb{Q})$ be the Hurewicz homomorphism. In 1965, Milnor and Moore [19] proved the following salient theorem (see also [18, page 293]).

Theorem 7. If $X$ is a simply connected topological space and if $\mathbb{F}$ is a field of characteristic zero, then

(1) the Samelson product makes $\pi_{*}(\Omega X) \otimes \mathbb{F}$ into a graded Lie algebra denoted by $L_{X}$;

(2) the Hurewicz homomorphism for $\Omega X$ is an isomorphism of $L_{X}$ onto the Lie algebra $P_{*}(\Omega X ; \mathbb{F})$ of primitive elements in $H_{*}(\Omega X ; \mathbb{F})$;

(3) the Hurewicz homomorphism extends to an isomorphism of graded Hopf algebras $U L_{X} \cong H_{*}(\Omega X ; \mathbb{F})$, where $U L_{X}$ is the universal enveloping algebra of $L_{X}$.

It is natural to ask what are the rationally nontrivial indecomposable and decomposable generators of the graded Lie algebra for $\Omega \Sigma B U(1)$ ? The following gives an answer to this question.

Theorem 8. The connected graded Lie algebra for $\Omega \Sigma B U(1)$ with the Samelson products modulo torsions is as follows:

$$
\frac{\pi_{*}(\Omega \Sigma B U(1))}{\text { torsion }}=L\left\{\xi_{1}, \xi_{2}, \ldots, \xi_{n}, \ldots\right\},
$$

where the dimension of $\xi_{n}$ is equal to $2 n$ in the graded homotopy groups for each $n=1,2,3, \ldots$.

Proof. It suffices to show that the iterated Samelson products $\left\langle\xi_{i_{k}},\left\langle\xi_{i_{k-1}}, \ldots,\left\langle\xi_{i_{1}}, \xi_{i_{2}}\right\rangle \cdots\right\rangle\right\rangle$ in homotopy groups $\pi_{2\left(i_{1}+\cdots+i_{k}\right)}(\Omega \Sigma B U(1))$ are rationally nontrivial decomposable generators, where the $\xi_{i_{j}}$ are indecomposable generators in dimension $2 i_{j}$, for $i_{j}=1,2,3, \ldots$ and $k \geq 2$.

We first note that the Eckmann-Hilton dual of the HopfThom theorem (see [11, pages 263-269] and [12, Chapter III]) says that $\Sigma B U(1)$ has the rational homotopy type of the wedge products of infinitely many spheres; that is,

$$
\Sigma B U(1) \simeq_{\mathbb{Q}} S^{3} \vee S^{5} \vee S^{7} \vee \cdots \vee S^{2 n+1} \vee \cdots .
$$

Let $\widehat{\xi}_{i_{j}}: S^{2 i_{j}+1} \rightarrow \Sigma B U(1)$ be the adjoint of $\xi_{i_{j}}: S^{2 i_{j}} \rightarrow$ $\Omega \Sigma B U(1)$. We prove the result in the case of twofold Samelson products. Suppose that $\left\langle\xi_{i_{1}}, \xi_{i_{2}}\right\rangle$ is rationally trivial in
$\pi_{2\left(i_{1}+i_{2}\right)}(\Omega \Sigma B U(1))$. It follows by the adjointness and the Hilton's formula that the twofold Whitehead products $\left[\widehat{\xi}_{i_{1}}\right.$, $\left.\widehat{\xi}_{i_{2}}\right]$ have a finite order in $\pi_{2\left(i_{1}+i_{2}\right)+1}\left(S^{2 i_{1}+1} \vee S^{2 i_{2}+1}\right)$ which is a subgroup of $\pi_{2\left(i_{1}+i_{2}\right)+1}(\Sigma B U(1))$. By using a cofibration sequence

$$
\begin{aligned}
& S^{2\left(i_{1}+i_{2}\right)+1} \stackrel{\left[\hat{\xi}_{i_{1}}, \hat{\xi}_{i_{2}}\right]}{\longrightarrow} S^{2 i_{1}+1} \vee S^{2 i_{2}+1} \longrightarrow S^{2 i_{1}+1} \times S^{2 i_{2}+1} \\
& \longrightarrow S^{2 i_{1}+1} \wedge S^{2 j_{1}+1} \longrightarrow \cdots
\end{aligned}
$$

we have

$$
S^{2 i_{1}+1} \times S^{2 i_{2}+1} \simeq\left(S^{2 i_{1}+1} \vee S^{2 i_{2}+1}\right) \cup_{\left[\widehat{\xi}_{i_{1}}, \widehat{\xi}_{i_{2}}\right]} e^{2\left(i_{1}+i_{2}\right)+2},
$$

where $\left[\widehat{\xi}_{i_{1}}, \widehat{\xi}_{i_{2}}\right]: S^{2\left(i_{1}+i_{2}\right)+1} \rightarrow S^{2 i_{1}+1} \vee S^{2 i_{2}+1}$ is the attaching map. The assumption shows that $\left[\widehat{\xi}_{i_{1}}, \widehat{\xi}_{i_{2}}\right]_{\mathbb{Q}} \simeq *$ when $\left[\widehat{\xi}_{i_{1}}, \widehat{\xi}_{i_{2}}\right]$ is rationalized, and thus

$$
S^{2 i_{1}+1} \times S^{2 i_{2}+1} \simeq_{\mathbb{Q}} S^{2 i_{1}+1} \vee S^{2 i_{2}+1} \vee S^{2\left(i_{1}+i_{2}\right)+2}
$$

where " $\simeq$ " is a rational homotopy equivalence. We also have a contradiction by applying the cohomology cup products to the above rational homotopy equivalence.

For induction, we now suppose that the $(k-1)$-fold Samelson products $\left\langle\xi_{i_{k-1}}, \ldots,\left\langle\xi_{i_{1}}, \xi_{i_{2}}\right\rangle \cdots\right\rangle$ are rationally nontrivial in the homotopy group $\pi_{2\left(i_{1}+\cdots+i_{k-1}\right)}(\Omega \Sigma B U(1))$. By Theorem 3 and adjointness again, we can consider the iterated Whitehead product $\left[\widehat{\xi}_{i_{k-1}}, \ldots,\left[\widehat{\xi}_{i_{1}}, \widehat{\xi}_{i_{2}}\right] \cdots\right]$ as a rational generator of

$$
\begin{array}{r}
\pi_{2\left(i_{1}+\cdots+i_{k-1}\right)+1}\left(S^{2\left(i_{1}+\cdots+i_{k-1}\right)+1}\right) \otimes \mathbb{Q} \\
\subset \pi_{2\left(i_{1}+\cdots+i_{k-1}\right)+1}(\Sigma B U(1)) \otimes \mathbb{Q} .
\end{array}
$$

Similarly, a cofibration shows that

$$
\begin{aligned}
& S^{2\left(i_{1}+\cdots+i_{k-1}\right)+1} \times S^{2 i_{k}+1} \\
& \quad \simeq\left(S^{2\left(i_{1}+\cdots+i_{k-1}\right)+1} \vee S^{2 i_{k}+1}\right) \cup_{\zeta} e^{2\left(i_{1}+\cdots+i_{k-1}+i_{k}\right)+2},
\end{aligned}
$$

where $\zeta=\left[\widehat{\xi}_{i_{k}},\left[\widehat{\xi}_{i_{k-1}}, \ldots,\left[\widehat{\xi}_{i_{1}}, \widehat{\xi}_{i_{2}}\right] \cdots\right]\right]: S^{2\left(i_{1}+\cdots+i_{k}\right)+1} \rightarrow$ $S^{2\left(i_{1}+\cdots+i_{k-1}\right)+1} \vee S^{2 i_{k}+1}$ is the attaching map. If this map has a finite order in homotopy groups, then we have a contradiction again by the same argument of the cohomology cup products.

Finally by taking the adjointness, we complete the proof.

Let $\bar{\xi}_{n}: S^{2 n} \rightarrow \Omega \Sigma B U(1)_{\mathbb{Q}}$ be the composition $r \circ \xi_{n}$ of the rationally nontrivial indecomposable element $\xi_{n}: S^{2 n} \rightarrow$ $\Omega \Sigma B U(1)$ of $\pi_{2 n}(\Omega \Sigma B U(1))$ with the rationalization $r$ : $\Omega \Sigma B U(1) \rightarrow \Omega \Sigma B U(1)_{\mathbb{Q}}$ for each $n=1,2, \ldots$. Since there is a one-to-one correspondence between the integral generators of homotopy groups modulo torsions and rational generators of rational homotopy groups; that is, $\operatorname{rank}_{\mathbb{Z}}\left(\pi_{2 n+1}(\Sigma B U(1)) /\right.$ torsion $)=\operatorname{rank}_{\mathbb{Q}}\left(\pi_{2 n+1}(\Sigma B U(1)) \otimes \mathbb{Q}\right)$, by using the MilnorMoore theorem and Theorem 8 , we have the following.

Corollary 9. The graded rational homotopy group $\pi_{*}(\Omega \Sigma B U(1)) \otimes \mathbb{Q}$ with the Samelson products becomes 
a connected graded free Lie algebra generated by $\left\{\bar{\xi}_{1}, \bar{\xi}_{2}, \ldots, \bar{\xi}_{n}\right.$, ...\}; that is,

$$
\pi_{*}(\Omega \Sigma B U(1)) \otimes \mathbb{Q} \cong L\left\{\bar{\xi}_{1}, \bar{\xi}_{2}, \ldots, \bar{\xi}_{n}, \ldots\right\}
$$

We note that the iterated Samelson products $\left\langle\bar{\xi}_{i_{k}},\left\langle\bar{\xi}_{i_{k-1}}\right.\right.$, $\left.\left.\ldots,\left\langle\bar{\xi}_{i_{1}}, \bar{\xi}_{i_{2}}\right\rangle \cdots\right\rangle\right\rangle$ are decomposable generators in the above free Lie algebra.

It is well known that the Hurewicz homomorphism $h$ : $\pi_{*}(\Omega \Sigma B U(1)) \rightarrow H_{*}(\Omega \Sigma B U(1) ; \mathbb{Q})$ carries the Samelson product into the Lie bracket defined by

$$
[z, w]=z w-(-1)^{|z||w|} w z
$$

where $z, w \in H_{*}(\Omega \Sigma B U(1) ; \mathbb{Q})$, and the multiplication is the Pontrjagin multiplication.

Theorem 10. Let $C\left({\widehat{F} i_{1}, F_{i_{2}}}\right): B U(1) \rightarrow \Omega \Sigma B U(1)$ be the adjoint of $C\left(F_{i_{1}}, F_{i_{2}}\right): \Sigma B U(1) \rightarrow \Sigma B U(1)$. Then one has the following:

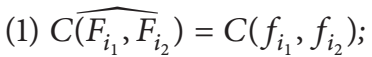

(2) $C\left(f_{i_{1}}, f_{i_{2}}\right)_{*}\left(u_{i_{1}+i_{2}}\right)=\left[h\left(\xi_{i_{1}}\right), h\left(\xi_{i_{2}}\right)\right]$, where $u_{i_{1}+i_{2}}$ is a rational homology generator in dimension $2\left(i_{1}+i_{2}\right)$, $\left[h\left(\xi_{i_{1}}\right), h\left(\xi_{i_{2}}\right)\right]$ is the Lie bracket, and $\xi_{i_{1}}$ and $\xi_{i_{2}}$ are homotopy elements of $\pi_{*}(\Omega \Sigma B U(1))$.

Proof. (1) The adjointness shows that, for $F \in[\Sigma B U(1)$, $\Sigma B U(1)], s \in B U(1), t \in I$, and $\langle s, t\rangle \in \Sigma B U(1)$, the map

$$
\kappa={ }^{-}:[\Sigma B U(1), \Sigma B U(1)] \longrightarrow[B U(1), \Omega \Sigma B U(1)],
$$

defined by

$$
(\kappa F)(s)(t)=\widehat{F}(s)(t)=F(\langle s, t\rangle),
$$

is a group isomorphism. It thus follows that

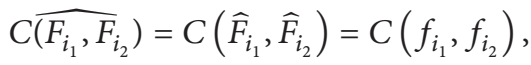

which is the commutator of $f_{i_{1}}$ and $f_{i_{2}}$ in $[B U(1), \Omega \Sigma B U(1)]$.

(2) For the second part, if $p: B U(1)_{2\left(i_{1}+i_{2}\right)} \rightarrow S^{2 i_{1}+2 i_{2}}$ is the projection map to the top cell and if $\langle$,$\rangle is the Samelson$ product in $\pi_{*}(\Omega \Sigma B U(1))$, then the following diagram is commutative up to homotopy (see also [20, Theorem 1.4]):

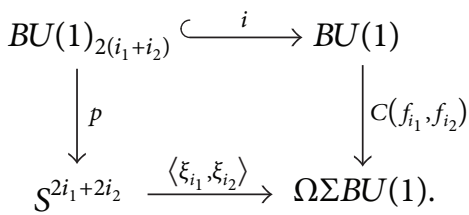

By applying the rational homology to the above diagram, we have

$$
\begin{aligned}
C\left(f_{i_{1}}, f_{i_{1}}\right)_{*}\left(u_{\left(i_{1}+i_{2}\right)}\right) & =C\left(f_{i_{1}}, f_{i_{1}}\right)_{*} i_{*}\left(u_{\left(i_{1}+i_{2}\right)}\right) \\
& =\left\langle\xi_{i_{1}}, \xi_{i_{2}}\right\rangle_{*} p_{*}\left(u_{\left(i_{1}+i_{2}\right)}\right) \\
& =\left\langle\xi_{i_{1}}, \xi_{i_{2}}\right\rangle_{*}\left(u_{\left(i_{1}+i_{2}\right)}^{\prime}\right) \\
& =h\left(\left\langle\xi_{i_{1}}, \xi_{i_{2}}\right\rangle\right) \\
& =\left[h\left(\xi_{i_{1}}\right), h\left(\xi_{i_{2}}\right)\right] .
\end{aligned}
$$

Here,

(i) $u_{i_{1}+i_{2}}$ is also used as a generator of

$$
H_{2\left(i_{1}+i_{2}\right)}\left(B U(1)_{2\left(i_{1}+i_{2}\right)} ; \mathbb{Q}\right) \cong H_{2\left(i_{1}+i_{2}\right)}(B U(1) ; \mathbb{Q}) ;
$$

(ii) $i_{*}\left(u_{\left(i_{1}+i_{2}\right)}\right)=u_{\left(i_{1}+i_{2}\right)}$;

(iii)

$$
p_{*}: H_{2\left(i_{1}+i_{2}\right)}\left(B U(1)_{2\left(i_{1}+i_{2}\right)} ; \mathbb{Q}\right) \longrightarrow H_{2\left(i_{1}+i_{2}\right)}\left(S^{2\left(i_{1}+i_{2}\right)} ; \mathbb{Q}\right)
$$

is an isomorphism sending the generator $u_{\left(i_{1}+i_{2}\right)}$ to the fundamental homology class $u_{\left(i_{1}+i_{2}\right)}^{\prime}$.

Theorem 11. Let $E: B U(1) \rightarrow \Omega \Sigma B U(1)$ be the canonical inclusion and let $p_{i_{1}+i_{2}}: B U(1) \rightarrow B U(1) / B U(1)_{2 i_{1}+2 i_{2}-1}$ be the projection map. Then for a given commutator $C\left(F_{i_{1}}, F_{i_{2}}\right)$ : $\Sigma B U(1) \rightarrow \Sigma B U(1)$, there exists a map

$$
\overline{\Omega C\left(F_{i_{1}}, F_{i_{2}}\right) \circ E}: \frac{B U(1)}{B U(1)_{2 i_{1}+2 i_{2}-1}} \longrightarrow \Omega \Sigma B U(1)
$$

such that $\overline{\Omega C\left(F_{i_{1}}, F_{i_{2}}\right) \circ E} \circ p_{i_{1}+i_{2}}=\Omega C\left(F_{i_{1}}, F_{i_{2}}\right) \circ E$.

Proof. We first show that the following diagram is strictly commutative:

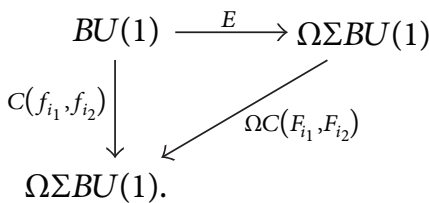

Indeed, the composition $\Omega C\left(F_{i_{1}}, F_{i_{2}}\right) \circ E: B U(1) \rightarrow$ $\Omega \Sigma B U(1)$ induces a map

$$
\left(\Omega C\left(F_{i_{1}}, F_{i_{2}}\right) \circ E\right)(s): I \longrightarrow \Sigma B U(1)
$$

sending $t \in I$ to

$$
\left(\Omega C\left(F_{i_{1}}, F_{i_{2}}\right) \circ E\right)(s)(t)=C\left(F_{i_{1}}, F_{i_{2}}\right)(\langle s, t\rangle) .
$$


On the other hand, $C\left(f_{i_{1}}, f_{i_{2}}\right): B U(1) \rightarrow \Omega \Sigma B U(1)$ sends $s \in B U(1)$ to

$$
C\left(f_{i_{1}}, f_{i_{2}}\right)(s): I \longrightarrow \Sigma B U(1) .
$$

By adjointness, we have

$$
\begin{aligned}
\left(C\left(f_{i_{1}}, f_{i_{2}}\right)(s)\right)(t) & \left.=\left(C \widehat{\left(F_{i_{1}}, F_{i_{2}}\right.}\right)(s)\right)(t) \\
& =C\left(F_{i_{1}}, F_{i_{2}}\right)(\langle s, t\rangle),
\end{aligned}
$$

where $\langle s, t\rangle$ is an element of $\Sigma B U(1)$. Therefore, we get

$$
\Omega C\left(F_{i_{1}}, F_{i_{2}}\right) \circ E=C\left(f_{i_{1}}, f_{i_{2}}\right) .
$$

We now consider the cell structure on $B U(1) \times B U(1)$ with

$$
(B U(1) \times B U(1))_{2 i_{1}+2 i_{2}-1}=\bigcup_{m+n=2 i_{1}+2 i_{2}-1} B U(1)_{m} \times B U(1)_{n} .
$$

Since the restrictions $\left.f_{i_{1}}\right|_{B U(1)_{2 i_{1}-1}}$ and $\left.f_{i_{2}}\right|_{B U(1)_{2 i_{2}-1}}$ are inessential from our construction of $f_{n}: B U(1) \rightarrow$ $\Omega \Sigma B U(1)$ for each $n=1,2,3, \ldots$, we see that if $m \leq 2 i_{1}-1$ or $n \leq 2 i_{2}-1$, then $\left.c\left(f_{i_{1}}, f_{i_{2}}\right)\right|_{B U(1)_{m} \times B U(1)_{n}}$ is null homotopic. By using the homotopy extension property, we can extend the null homotopy to all of $(B U(1) \times B U(1))_{2 i_{1}+2 i_{2}-1}$. Thus, by the cellular approximation theorem, we have

$$
\begin{aligned}
\left.\Omega C\left(F_{i_{1}}, F_{i_{2}}\right) \circ E\right|_{B U(1)_{2 i_{1}+2 i_{2}-1}} & =\left.C\left(f_{i_{1}}, f_{i_{2}}\right)\right|_{B U(1))_{2 i_{1}+2 i_{2}-1}} \\
& =\left.c\left(f_{i_{1}}, f_{i_{2}}\right) \circ \Delta\right|_{B U(1)_{2 i_{1}+2 i_{2}-1}} \\
& \left.\simeq c\left(f_{i_{1}}, f_{i_{2}}\right) \circ \Delta^{\prime}\right|_{B U(1)_{2 i_{1}+i_{2}-1}} \\
& \simeq *,
\end{aligned}
$$

where $\Delta^{\prime}: B U(1) \rightarrow B U(1) \times B U(1)$ is a cellular map. Therefore there exists a map

$$
\overline{\Omega C\left(F_{i_{1}}, F_{i_{2}}\right) \circ E}: \frac{B U(1)}{B U(1)_{2 i_{1}+2 i_{2}-1}} \longrightarrow \Omega \Sigma B U(1)
$$

such that the following diagram is commutative up to homotopy:

$$
B U(1)_{2\left(i_{1}+i_{2}\right)-1} \underset{\Omega C\left(F_{i_{1}}, F_{i_{2}}\right) \circ E \underbrace{}_{\Omega \Sigma B U(1)} \stackrel{i_{i_{1}+i_{2}}}{\longrightarrow}}{B U(1) \stackrel{p_{i_{1}+i_{2}}}{\longrightarrow} B U(1) / B U(1)_{2\left(i_{1}+i_{2}\right)-1}}
$$

where the top row is a cofibration sequence as required.

The Milnor-Moore theorem asserts that the image of the Hurewicz homomorphism is primitive. The following is another expression of the primitive elements in the Pontrjagin algebra.
Theorem 12. The image of a homomorphism

$$
f_{n_{*}}: H_{2 n}(B U(1)) \longrightarrow H_{2 n}(\Omega \Sigma B U(1))
$$

is primitive for each $n=1,2,3, \ldots$.

Proof. Since $f_{n}: B U(1) \rightarrow \Omega \Sigma B U(1), n=1,2,3, \ldots$ can be factored as

$$
B U(1) \stackrel{p_{n}}{\longrightarrow} \frac{B U(1)}{B U(1)_{2 n-1}} \stackrel{\tilde{f}_{n}}{\longrightarrow} \Omega \Sigma B U(1)
$$

so that the restriction to the bottom sphere of the map $\tilde{f}_{n}$ coincides with the map $\xi_{n}: S^{2 n} \rightarrow \Omega \Sigma B U(1)$, and since $B U(1) / B U(1)_{2 n-1}$ is $(2 n-1)$-connected, by the Hurewicz isomorphism theorem, every class in $H_{2 n}\left(B U(1) / B U(1)_{2 n-1}\right)$ is spherical, and thus primitive. We therefore know that the image of $\tilde{f}_{n_{*}}$ lies in the set of primitives $P_{2 n}(\Omega \Sigma B U(1))$ in $H_{2 n}(\Omega \Sigma B U(1))$, so does the image of $f_{n_{*}}$ for each $n=$ $1,2,3, \ldots$.

Since the restriction to the skeleton

$$
\left.\Omega C\left(F_{i_{k}}, C\left(F_{i_{k-1}}, \ldots, C\left(F_{i_{1}}, F_{i_{2}}\right) \ldots\right)\right) \circ E\right|_{B U(1)_{2\left(i_{1}+i_{2}+\cdots+i_{k}\right)-1}}
$$

is null homotopic, by Theorem 12, we have the following.

\section{Corollary 13.}

$$
\Omega C\left(F_{i_{k}}, C\left(F_{i_{k-1}}, \ldots, C\left(F_{i_{1}}, F_{i_{2}}\right) \cdots\right)\right)_{*}\left(u_{i_{1}+i_{2}+\cdots+i_{k}}\right)
$$

is in the Lie subalgebra $P_{*}(\Omega \Sigma B U(1) ; \mathbb{Q})$ of primitive elements in $H_{*}(\Omega \Sigma B U(1) ; \mathbb{Q})$.

Remark 14. We note that the self-map

$$
\begin{aligned}
& \Omega C\left(F_{i_{k}}, C\left(F_{i_{k-1}}, \ldots, C\left(F_{i_{1}}, F_{i_{2}}\right) \ldots\right)\right): \Omega \Sigma B U(1) \\
& \longrightarrow \Omega \Sigma B U(1)
\end{aligned}
$$

is a loop map; thus it is an H-map. It is well known in [3, page 75] that there is a bijection between the groups $[\Sigma B U(1), \Sigma B U(1)]$ and $[\Omega \Sigma B U(1), \Omega \Sigma B U(1)]_{H}$ of homotopy classes of H-maps $\Omega \Sigma B U(1) \rightarrow \Omega \Sigma B U(1)$. We thus get the corresponding self-map $C\left(F_{i_{k}}, C\left(F_{i_{k-1}}, \ldots, C\left(F_{i_{1}}, F_{i_{2}}\right) \cdots\right)\right)$ in the group $[\Sigma B U(1), \Sigma B U(1)]$. Moreover, by using the classical Whitehead theorem, we obtain a homotopy self-equivalence of the form $I+C\left(F_{i_{k}}, C\left(F_{i_{k-1}}, \ldots, C\left(F_{i_{1}}, F_{i_{2}}\right) \cdots\right)\right)$; that is, $I+$ $C\left(F_{i_{k}}, C\left(F_{i_{k-1}}, \ldots, C\left(F_{i_{1}}, F_{i_{2}}\right) \cdots\right)\right) \in \operatorname{Aut}(\Sigma B U(1))$, where $I$ is the identity map and "+" is the suspension addition on $\Sigma B U(1)$.

\section{Acknowledgments}

The author is grateful to an anonymous referee for a careful reading and many helpful suggestions that improved the quality of the paper. This research was supported by Basic Science Research Program through the National Research Foundation of Korea (NRF) funded by the Ministry of Education, Science and Technology (2012-0007611). 


\section{References}

[1] J. D. Stasheff, "Homotopy associativity of $H$-spaces. I, II," Transactions of the American Mathematical Society, vol. 108, pp. 275-312, 1963.

[2] M. Arkowitz, "Co-H-spaces," in Handbook of Algebraic Topology, pp. 1143-1173, North-Holland, Amsterdam, The Netherlands, 1995.

[3] H. Scheerer, "On rationalized $H$ - and co- $H$-spaces. With an appendix on decomposable $H$ - and co- $H$-spaces," Manuscripta Mathematica, vol. 51, no. 1-3, pp. 63-87, 1985.

[4] J. F. Adams, "An example in homotopy theory", Mathematical Proceedings of the Cambridge Philosophical Society, vol. 53, pp. 922-923, 1957.

[5] B. I. Gray, "Spaces of the same $n$-type, for all n," Topology, vol. 5, pp. 241-243, 1966.

[6] D.-W. Lee, "On the same $n$-type structure for the suspension of the Eilenberg-Mac Lane spaces," Journal of Pure and Applied Algebra, vol. 214, no. 11, pp. 2027-2032, 2010.

[7] C. A. McGibbon and J. M. Møller, "On spaces with the same n-type for all n," Topology, vol. 31, no. 1, pp. 177-201, 1992.

[8] C. A. McGibbon, "Self-maps of projective spaces," Transactions of the American Mathematical Society, vol. 271, no. 1, pp. 325346, 1982.

[9] M. A. Kutbi, J. Ahmad, N. Hussain, and M. Arshad, "Common fixed point results for mappings with rational expressions," Abstract and Applied Analysis, vol. 2013, Article ID 549518, 11 pages, 2013.

[10] S. M. Abusalim and M. S. M. Noorani, "Fixed point and common fixed point theorems on ordered cone $b$-metric spaces," Abstract and Applied Analysis, vol. 2013, Article ID 815289, 7 pages, 2013.

[11] E. H. Spanier, Algebraic Topology, McGraw-Hill, New York, NY, USA, 1966.

[12] G. W. Whitehead, Elements of Homotopy Theory, vol. 61 of Graduate Texts in Mathematics, Springer, New York, NY, USA, 1978.

[13] R. Bott and H. Samelson, "On the Pontryagin product in spaces of paths," Commentarii Mathematici Helvetici, vol. 27, pp. 320337, 1953.

[14] P. J. Hilton, "On the homotopy groups of the union of spheres," Journal of the London Mathematical Society, vol. 30, pp. 154-172, 1955.

[15] M. Arkowitz and C. R. Curjel, "Homotopy commutators of finite order. I," The Quarterly Journal of Mathematics, vol. 14, pp. 213-219, 1963.

[16] M. Arkowitz, “Commutators and cup products," Illinois Journal of Mathematics, vol. 8, pp. 571-581, 1964.

[17] F. R. Cohen, J. C. Moore, and J. A. Neisendorfer, "Torsion in homotopy groups," Annals of Mathematics, vol. 109, no. 1, pp. 121-168, 1979.

[18] Y. Félix, S. Halperin, and J.-C. Thomas, Rational Homotopy Theory, vol. 205 of Graduate Texts in Mathematics, Springer, New York, NY, USA, 2001.

[19] J. W. Milnor and J. C. Moore, "On the structure of Hopf algebras," Annals of Mathematics, vol. 81, pp. 211-264, 1965.

[20] K. Morisugi, "Projective elements in $K$-theory and self maps of $\Sigma \mathbb{C} P^{\infty}$," Journal of Mathematics of Kyoto University, vol. 38, no. 1, pp. 151-165, 1998. 


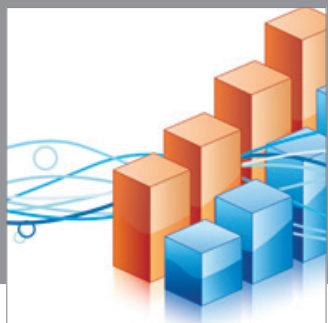

Advances in

Operations Research

mansans

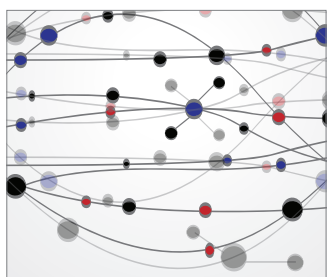

The Scientific World Journal
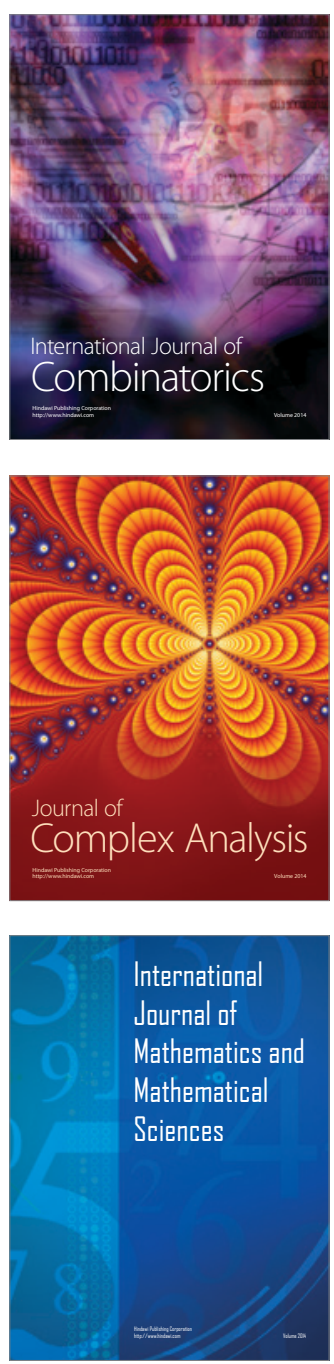
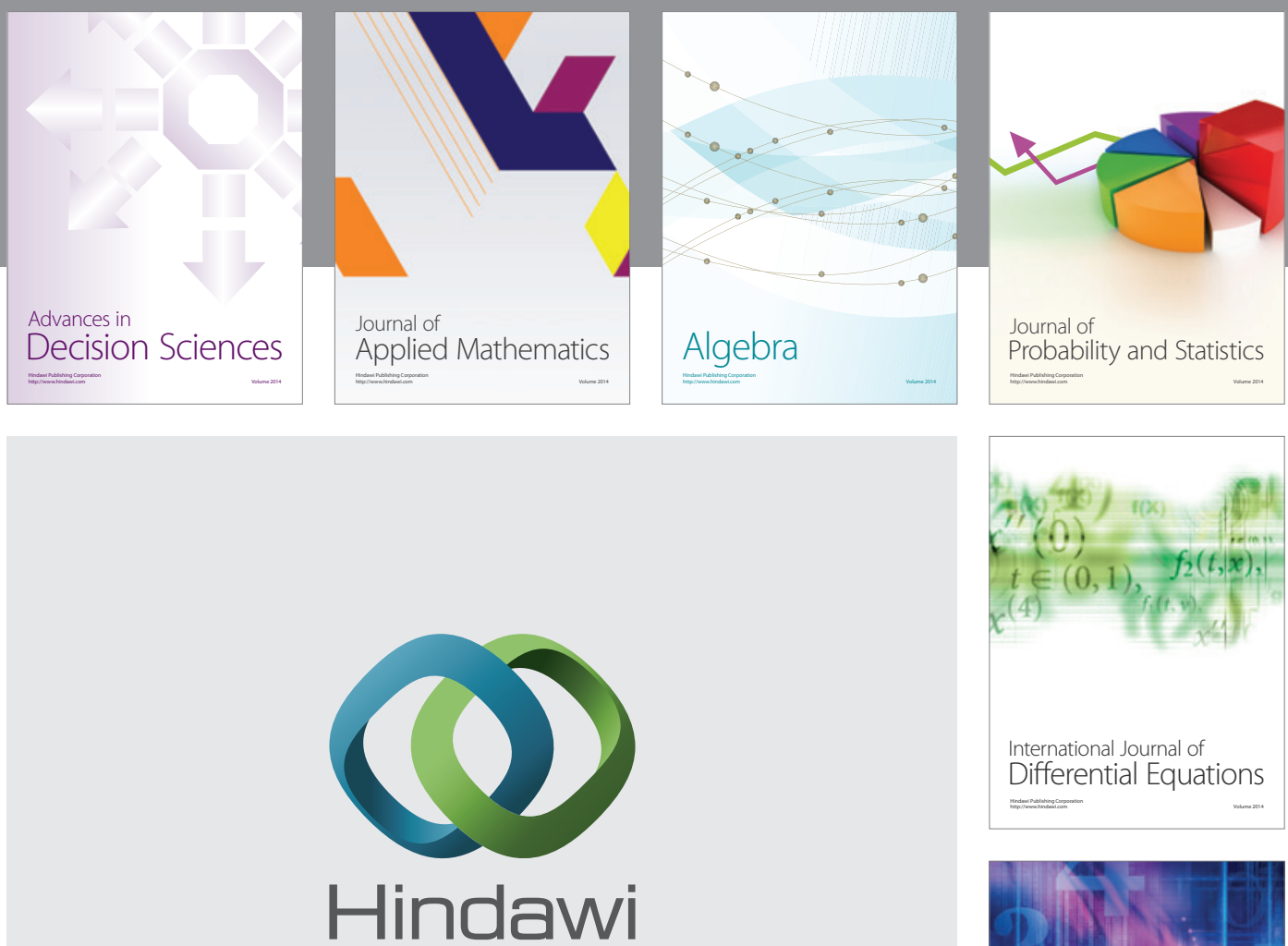

Submit your manuscripts at http://www.hindawi.com
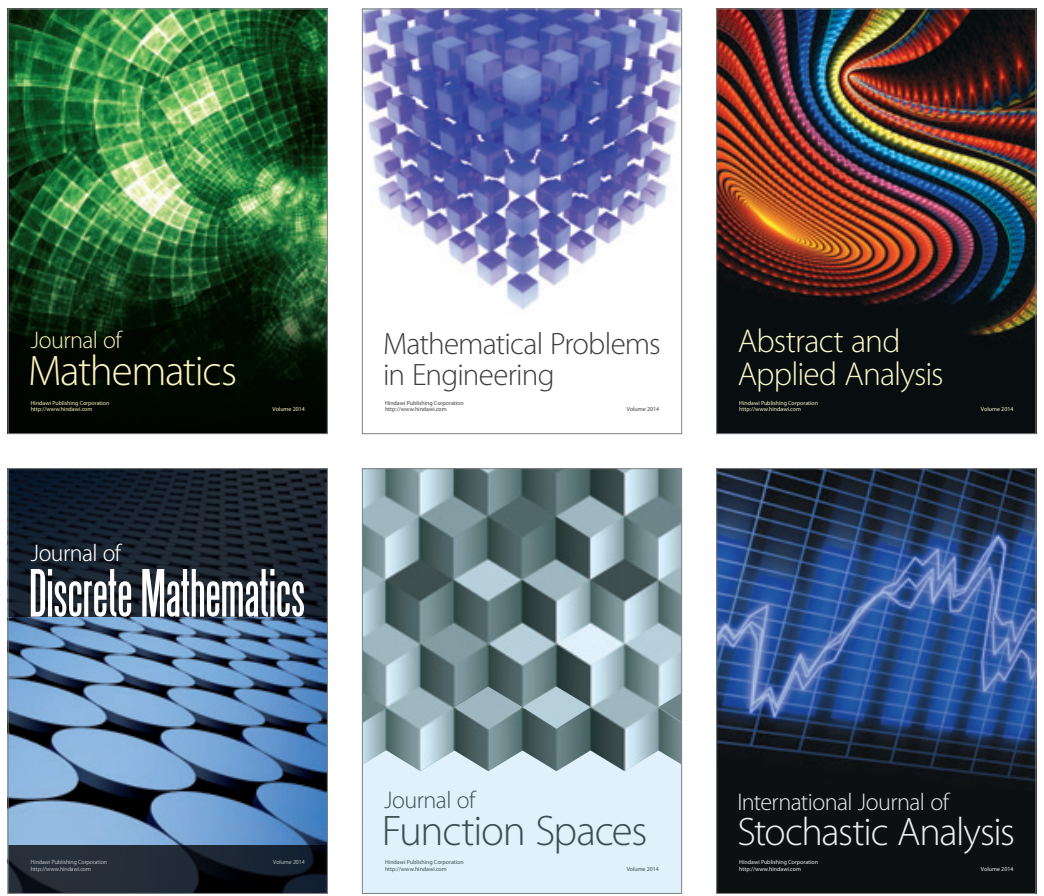

Journal of

Function Spaces

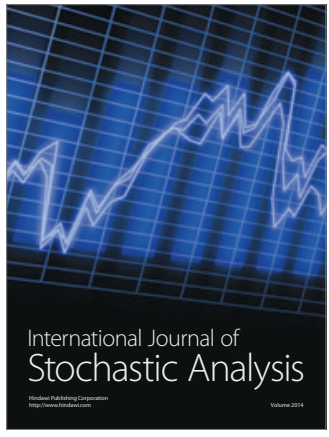

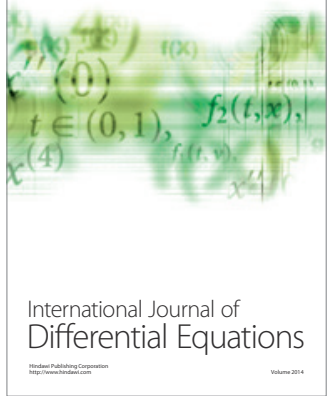
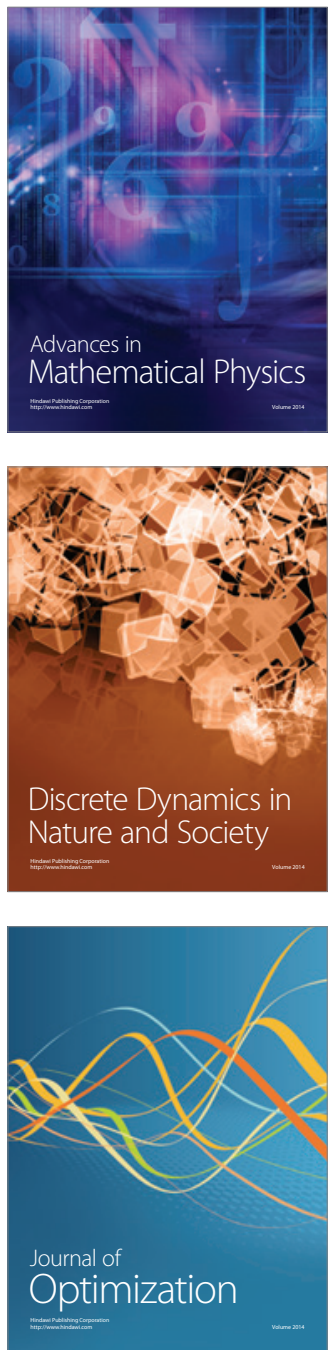